\title{
Simulation and Performance Analysis of Low Power Quasi Floating Gate PCS
}

\author{
Model \\ Pawan Whig ${ }^{1}$ \\ Syed Naseem Ahmad ${ }^{2}$ \\ ${ }^{I}$ Vivekananda Institute of Professional Studies, New Delhi, India \\ ${ }^{2}$ Jamia Millia Islamia ,New Delhi,India \\ * Corresponding author’s Email: pawanwhig@gmail.com, snahmad@jmi.ac.in
}

\begin{abstract}
The Spice Model for photo catalytic sensor (PCS) proposed by Whig and Ahmad overcome several drawbacks like complex designing, non-linearity and long computation time generally found in Flow Injection Analysis (FIA) technique by Yoon-Chang Kim et al for the determination of Chemical Oxygen Demand (COD). FIA technique involves the complete analysis including sampling and washing. Flow Injection Analysis is analytical method for the measurement of chemical oxygen demand using photochemical column. This method uses a bulky setup and takes 10-15 minutes to get the output result which is a tedious and time consuming job. If conventional method is continuously used for a long time than it is stable only for 15 days. The purpose of this paper is to propose a new Quasi Floating Gate Photo Catalytic Sensor (QFGPCS) approach which has low power consumption and compared the results with the conventional models. The proposed model operates under sub threshold conditions which are appreciated in large integrated system design. The results of simulation were found to be fairly in agreement with the theoretical predictions. The results are exhibited near linear variations of parameters of interest with appreciably reduced response time.
\end{abstract}

Keywords: QFGPCS, SPICE modeling, Simulation, Photo catalytic sensor, Flow injection analysis, Macro model.

\section{Introduction}

In the field of artificial waste water analysis and COD determination, $\mathrm{TiO}_{2}$ sensors have large number of potential applications due to their highly efficient photo activity, steadiness and least cost. From the earlier times $\mathrm{TiO}_{2}$ is used as white pigment which is safe for human use. The results achieved by the use of $\mathrm{TiO}_{2}$ Photo Catalytic Sensor are in good agreement with those from the conventional Dichromate method. Very complex and bulky set up is needed for the conventional method used for Photo Catalytic Sensor applications and consumes a plenty of time for computation. To provide a solution to these problems and make the application faster, Whig and Ahmad (2013) developed a Simulation Program with Integrated Circuit
Emphasis (SPICE) model for Photo Catalytic Sensor. With the advancement in semiconductor technology development of sensors has become easy due to advantages of low power, high speed signal processing capabilities. CAD tools are an added advantage as they provide a method for simulation and synthesis of semiconductor sensors. By using the SPICE model, the size and power of the overall system can be minimized thus increasing the reliability of the system.

The first floating gate (FG) device was reported by Sze in 1967 [1]. FG's are generally used in digital memory applications but recently some new applications of FG have been developed in which they are used as circuit elements. FG circuits are generally considered for systems requiring low power consumption and controlled second order effects as compared to other technologies. There is a 
recent trend towards utilizing $\mathrm{FG}$ to solve the practical problems associated with analog and digital circuits.

An FG is made up of a poly silicon gate which is covered by silicon dioxide. FG technology is used by Harrison and colleagues to remove the on chip biasing voltages. This is done by providing these voltages on-chip with arrays of programmable FG voltages [2]. FG technology is also used in advanced analog or mixed mode VLSI chips having large numbers of inputs and analog parameters. On chip technology reduces the complexity of the system due to negligible interconnection length. A new name called neuron MOS transistors had been given to FG devices by Shibata and Ohmi [3], due to analogy between the function performed by these devices and cells in the nervous system. These devices are referred as multiple input FGMOS by Yang and Andreou [4]. The concept of FGMOS transistors introduced by Shibata and Ohmi has resulted in a number of interesting analog and digital signal processing circuits, which also includes a multiple input FG, differential amplifier [5] and D/A converters.

There is a strict requirement of knowledge of code structure and sub routines for modeling the MOSFETs [6] and it is based on an appropriate SPICE version. This paper presents a SPICE macro model for FGPCS which has been implemented in Tanner tool. A large number of MOSFET models operating in sub threshold region are present in this tool. The basic model of FGPCS consists of an oxygen sensitive surface which has an insulator layer of Titanium Oxide $\left(\mathrm{TiO}_{2}\right)$ exposed to an electrolyte solution. When a $\mathrm{p}$-type semiconductor and $\mathrm{TiO}_{2}$ insulator are placed in an aqueous electrolyte solution the reaction (response) of FGPCS to $\mathrm{O}_{2}$ can be elucidated in terms of photo catalysis. At submicron level, gate oxide has very small thickness. As the dimension of gate oxide decreases the leakage currents of MOSFETS are increased due to tunnelling of the charge carriers which results in diminution of reliability of the device. For continuous reduction of the dimensions materials having much higher permittivity than silicon dioxide are used. The combination of Cassius-Mosetti equation with permittivity is given by Stephen et al. (1997). According to them, when permittivity of the dielectric increases, the band gap of dielectric material decreases resulting in tunnelling phenomenon [7]. Brown and Grannemann in 1978 studied that capacitance in accumulation is generally found to depend on gate bias. But as compared to Capacitance Voltage (CV) curve of $\mathrm{SiO}_{2}$ capacitor this effect is less pronounced in $\mathrm{TiO}_{2}$ capacitor sensor. From the $\mathrm{CV}$ measurements of $\mathrm{TiO}_{2}$ capacitor it was found that mid-gap interface state density was typically found to be between $3 \times$ $10^{10}$ and $10^{11} \mathrm{~V}^{-1} \mathrm{~cm}^{-2}$. It is described by Fuyuki and Matsunami (1986) that the magnitude of the interface state density for $\mathrm{TiO}_{2}$ capacitor is given by the product of conductance of $\mathrm{TiO}_{2}$ at fixed dc bias and peak conductance frequency. The width of accumulation layer is determined by the wave function of the Eigen states explained by (Duffy, 1990; Rausch and Burte, 1992). The solution of the Schrodinger equation at the interface leads to Airy function which produces an average position for accumulation of carriers in the ith energy state.

$$
E i \approx\left[\frac{h^{2}}{8 \pi^{2} m^{*}}\right]^{\frac{1}{3}}\left[\frac{3 \pi}{2} q F_{S}\left(i+\frac{3}{4}\right)\right]^{\frac{2}{3}}
$$

Where $\mathrm{q}$ is the electron charge, $\mathrm{F}_{\mathrm{s}}$ is the electric field at the surface, $E_{i}$ is the ith energy level, $m^{*}$ is the effective mass and $h$ is Plank's constant. For reasonable assumptions the accumulation layer thickness (x) should be of the order of $40 \AA$. Thus the tunnelling effect is small for $\mathrm{TiO}_{2}$ layers with thickness much greater than $15 \AA$. To consider this effect a series capacitance is added to $\mathrm{C}_{\mathrm{ox}}$ which is called quantum capacitance $\mathrm{C}_{\mathrm{q}}$ and therefore equivalent capacitance $C_{M}$ is given by

$$
\frac{1}{C_{M}}=\frac{1}{C_{q}}+\frac{1}{C_{o x}}=\frac{t_{T^{2 i o} o_{2}}}{A \varepsilon_{T_{i o} \varepsilon_{o}}}+\frac{\langle x\rangle}{A \varepsilon_{S i} \varepsilon_{o}}
$$

In this research study the simulation of Low Power Quasi Floating Gate PCS is presented further the result obtained is compared with previous PCS designs.

\section{Floating Gate PCS (FGPCS) Model}

The structure of FGPCS is similar to that of PCS model having gate terminal placed in the solution proposed by [8] Whig and Ahmad. The gate of the FGPCS is electrically isolated which creates a floating node in the DC, and a number of secondary gates called control gates are deposited above the floating gate which are electrically isolated. The voltage on the floating gate can be determined by the amount of charge deposited on it and the voltage on the multiple gates, source, drain and substrate which in turn modulates the channel conductivity [9].The structure of FGPCS is shown in Fig. 1. 


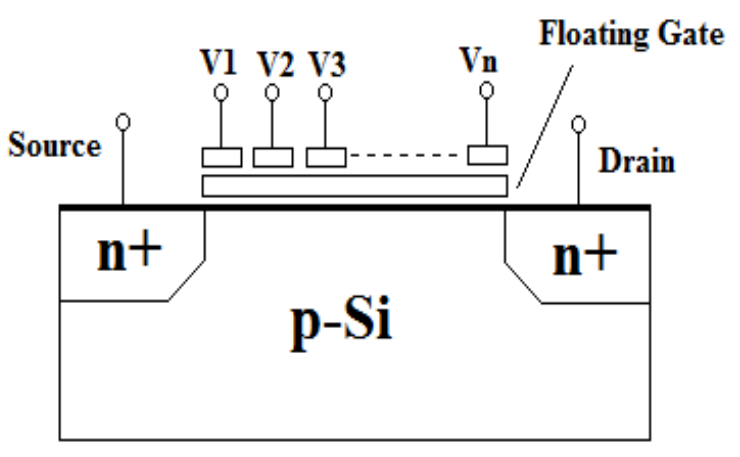

Multiple Input FGPCS

Figure.1 Cross section of FGPCS.

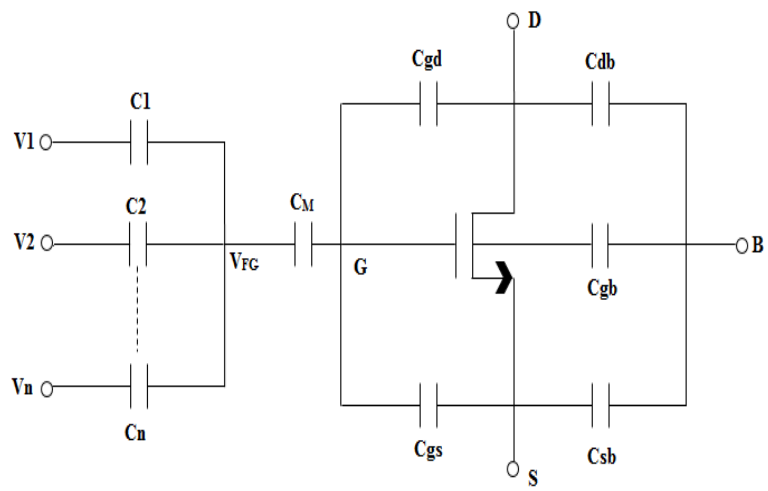

Figure.2 Circuit for FGPCS

The equivalent circuit for structure of FGPCS is shown in Fig.2. The FG of the structure is capacitive coupled to the inputs and the voltage present at these gates can modulate the channel current. The expression for the floating gate voltage $V_{F G}$ for FGPCS is given as:

$V \mathrm{FG}=\frac{C_{g d} V_{D}+C_{g s} V_{S}+\left(C_{g b}+C_{d b}+C_{s b}\right) V_{B}+q_{F G}+\sum_{i=1}^{N} C_{i} V_{i}}{C_{T o t a l}}$

Where

$\mathrm{C}_{\text {Total }}=$ Total parasitic capacitance

$\mathrm{V}_{\mathrm{FG}}=$ Floating gate voltage

$\mathrm{V}_{\mathrm{i}}=$ Voltage of ith control gate

$q_{F G}=$ Total charge on floating gate

$\mathrm{C}_{\mathrm{fgs}}=$ Capacitance between gate and source.

$\mathrm{C}_{\mathrm{fds}}=$ Capacitance between drain and source.

$\mathrm{C}_{\mathrm{fgd}}=$ Capacitance between gate and drain.

$\mathrm{C}_{\mathrm{fgb}}=$ Capacitance between gate and bulk.

$\mathrm{C}_{\mathrm{fdb}}=$ Capacitance between drain and bulk.

$\mathrm{V}_{\mathrm{S}}=$ Source Voltage

$\mathrm{V}_{\mathrm{D}}=$ Drain Voltage

$\mathrm{V}_{\mathrm{B}}=$ Bulk Voltage

Large number of striking features consisting of low-voltage and low-power are provided by FGPCS device. Both linear and non-linear functions can be implemented easily with it. Moreover, designers can add extra inputs to provide tunability to the circuit.

The FGPCS has a sensitive surface which is made up of an insulation layer like $\mathrm{TiO}_{2}$ shown in Fig.3. $\mathrm{TiO}_{2}$ is used because it has very good dielectric constant which makes it suitable to be used at deep submicron level. $\mathrm{TiO}_{2}$ beads are used to vary the COD concentration in the water and its properties like non photo-corrosive, nontoxic and capable of the photo oxidative destruction of most organic pollutants makes its very important and inexpensive photo catalyst. A gate terminal placed directly in an aqueous solution with the help of electrodes is considered to calculate the response of FGPCS. The response of the FGPCS to COD can be explained in terms of semiconductor photo catalysis.

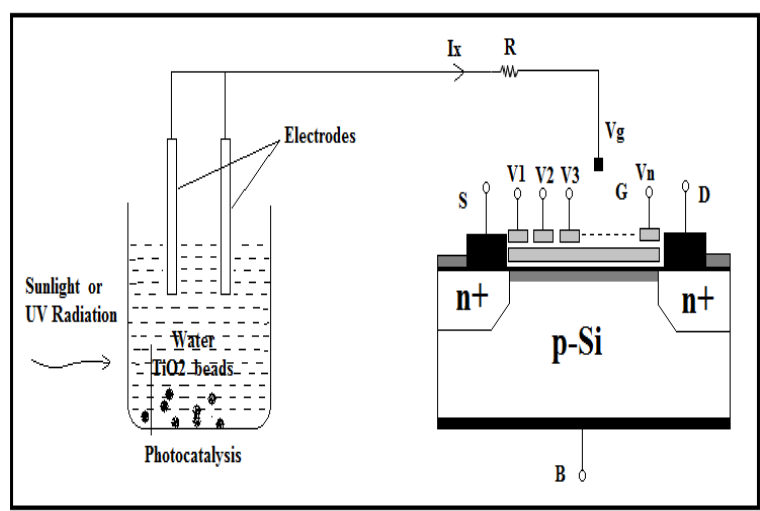

Figure.3 Sketch of PCS.

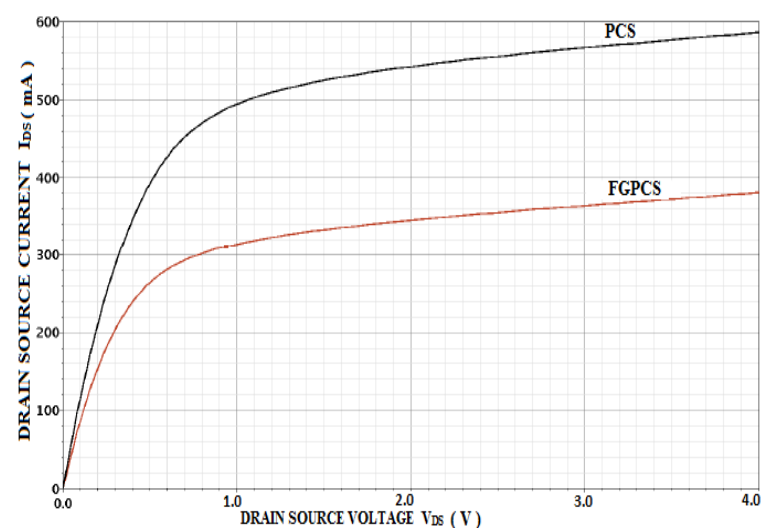

Figure.4 Characteristics curves for FGPCS and PCS

Due to the Photo Catalysis of $\mathrm{TiO}_{2}$ beads present in the water the current $I_{x}$ through the wire varies which in turn varies the gate voltage $\mathrm{V}_{\mathrm{g}}$ of FGPCS. As the photo catalysis occur, the maximum current decreases. It is observed that for a given sample 
when decrease in maximum current takes place due to photo catalysis there is corresponding decrease in the $V_{\mathrm{gs}}$ and drain current $\mathrm{I}_{\mathrm{ds}}$.

The output characteristics for the conventional PCS and FGPCS are shown in Fig 4. The curves are drawn between $I_{\mathrm{ds}}$ and $\mathrm{V}_{\mathrm{ds}}$ for PCS and FGPCS for $\mathrm{O}_{2}=1 \mathrm{mg} / \mathrm{L}$ with $\mathrm{E}_{\mathrm{ref}}=0$. It can be seen from the figure that current for FGPCS is lower than PCS at same voltage thus saving a power of about $30-40 \%$ and the threshold voltage for FGPCS is less than conventional PCS model.

However, the FGPCS model is not suitable because it gives low frequency response and requires large chip area. Moreover, due to isolated floating gate in FGPCS, static charge gets accumulated on it.

\section{Quasi Floating Gate PCS (QFGPCS) Model}

The structure of the QFGPCS is same as FGPCS model but the floating gate in QFGPCS is weakly connected to one of the supply rails through a high value of the resistor. The quasi floating gate of the PCS model is connected to the $V_{D D}$ through a reverse bias diode connected $\mathrm{P}$ type MOS which acts as a large value resistor. This pull up resistor sets the dc voltage at $\mathrm{Q}_{\mathrm{FG}}$ to either power rails and eliminates the power due to accumulated charge on the floating gate during fabrication process and also reduces the supply voltage requirements. The proposed structure of the QFGPCS is shown in Fig 5.

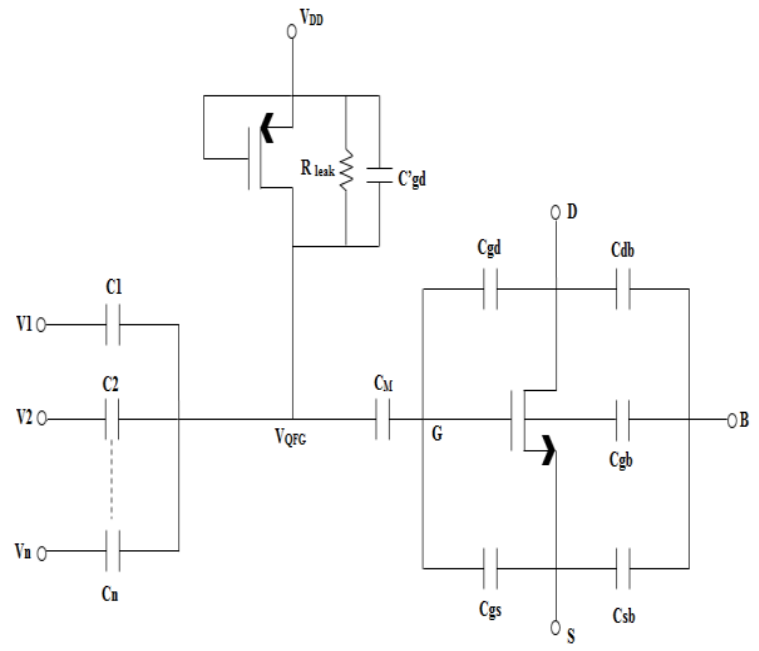

Figure.5 Proposed QFGPCS

The use of large resistance makes the quasi floating gate effectively floating for low frequency signals and ac operation of the signals of these frequency range remains unaffected.

\section{Mathematical Modeling}

A mathematical model is a description of a system using mathematical concepts and language. The process of developing a mathematical model is termed mathematical modeling. The parameters like quasi floating gate voltage, parasitic capacitances, leakage resistance, drain bulk and source voltage of the given system are used to describe mathematical model.

Various parasitic capacitance of device are considered to calculate total capacitance of the device. By considering total capacitance and leakage resistance the quasi floating gate voltage $\mathrm{V}_{\mathrm{QFG}}$ is given in eq. 4 :

$$
\begin{gathered}
V_{Q F G}=\left[C_{f g d} V_{D}+C_{f g s} V_{S}+\left(C_{f g b}+C_{f d b}+\right.\right. \\
\left.\left.C_{f s b}\right) V_{B}+\sum_{i=1}^{N} C_{i} V_{i}\right] * \frac{s R_{\text {leak }}}{1+s R_{\text {leak }} * C_{\text {total }}}
\end{gathered}
$$

Where,

$\mathrm{V}_{\mathrm{QFG}}=$ quasi floating gate voltage

$C_{\text {total }}=$ Total capacitance

$R_{\text {leak }}=$ Leakage Resistance

$V_{D}=$ Drain Voltage

$V_{S}=$ Source Voltage

$V_{B}=$ Bulk Voltage

The drain current for QFGPCS operating in linear region is given in Equation (5):

$$
I_{D}=\beta\left[\left(V_{Q F G}-V_{T}\right)-\frac{V_{D S}}{2}\right] V_{D S}
$$

Where,

$\beta=\mu_{n} C_{o x} \frac{W}{L}$

$\mu_{n} \quad=$ Mobility of charges

$C_{o x}=$ Oxide Capacitance

$V_{Q F G}=$ Quasi Floating Gate Voltage

$V_{T}=$ Threshold Voltage

$\frac{W}{L}=$ Width to length ratio

From Equation (4) it is observed that input signals encounter a high-pass filter with a cut-off frequency of $\frac{1}{2 \Pi R_{\text {leak }} C_{\text {total }}}$ which is very low due to large value of $R_{\text {leak }}$. Therefore, even for very low frequencies, Equation (4) becomes a weighted average of the AC input voltages determined by capacitance ratios plus some parasitic terms.

\section{Results \& Discussion}

The simulation of the device has been done on Tanner tool Version 15 for $90 \mathrm{~nm}$ technology. The 
developed device Model has been extensively tested and the simulation results have been compared with experimental data results of previous model. The relationship of $I_{d s}$ with $V_{g s}$ is shown in the Fig.6 which shows that proposed QFGPCS exhibits linear characteristic in the range of $\mathrm{V}_{\mathrm{gs}}=2.5 \mathrm{~V}$ to $5.0 \mathrm{~V}$.

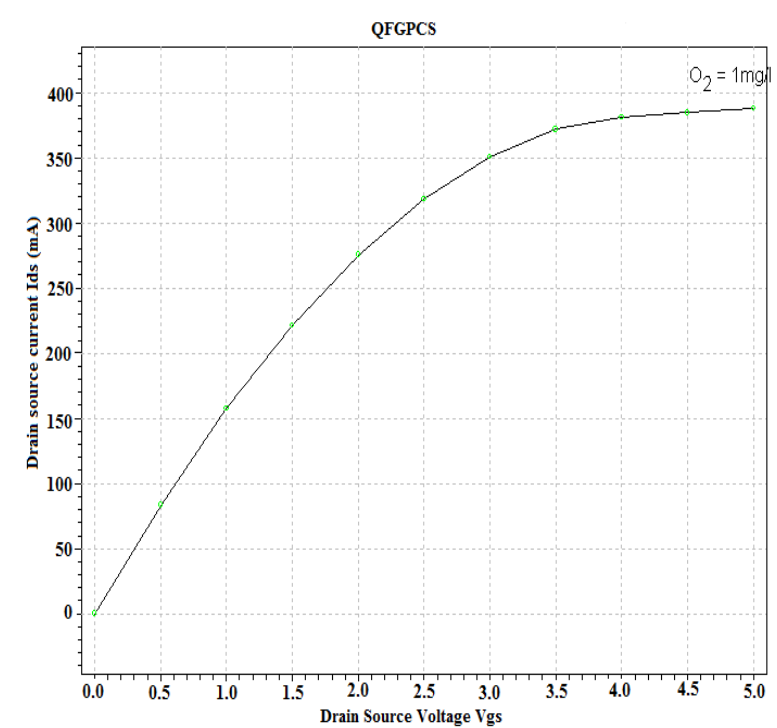

Figure.6 Characteristic curves between $\mathrm{I}_{\mathrm{ds}}$ and $\mathrm{V}_{\mathrm{gs}}$ at $\mathrm{O}_{2}=1-5 \mathrm{mg} / \mathrm{L}$ obtained through SPICE.

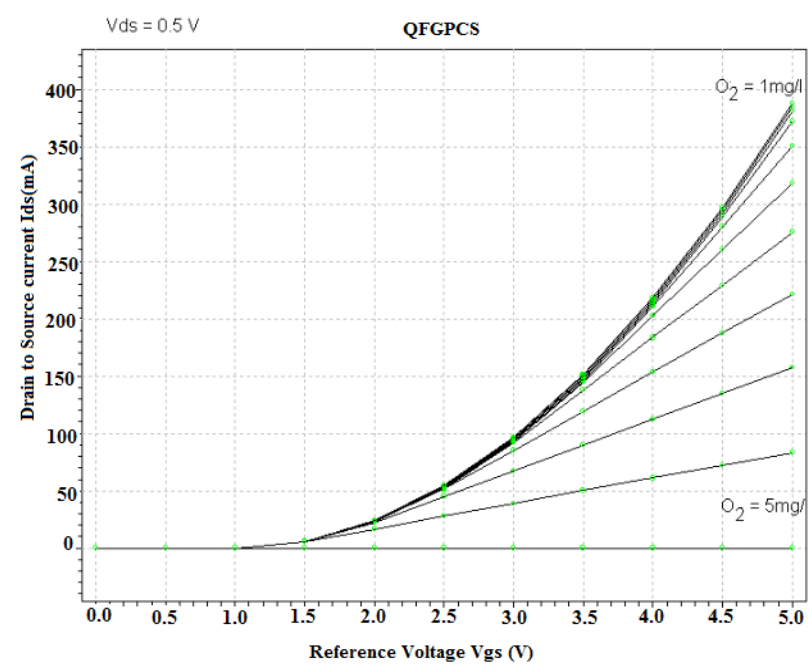

Figure. 7Characteristic curve for QFGPCS between $\mathrm{I}_{\mathrm{ds}}$ and $\mathrm{V}_{\mathrm{ds}}$ obtained through SPICE.

The curves are drawn between $\mathrm{I}_{\mathrm{ds}}$ and $\mathrm{V}_{\mathrm{gs}}$ of QFGPCS for $\mathrm{V}_{\mathrm{ds}}=0.5 \mathrm{~V}$ it can be seen that the proposed QFGPCS is fairly linear for concentrations more than $1 \mathrm{mg} / \mathrm{L}$ extending towards $5 \mathrm{mg} / \mathrm{L}$ of $\mathrm{O}_{2}$. This is an obvious advantage of this QFGPCS model.

By observation of Fig.7 it can be seen that the graph of $\mathrm{I}_{\mathrm{ds}} \mathrm{Vs} \mathrm{V}_{\mathrm{gs}}$ using QFGPCS model is fairly matching the plot obtained for the experimental data where $\mathrm{I}_{\mathrm{ds}}$ depends on $\Delta \mathrm{I}$ and $\mathrm{V}_{\mathrm{ds}}$ depends on $\mathrm{O}_{2}$ concentration. The comparison validates the
QFGPCS model's working. The linear variation of $\mathrm{I}_{\mathrm{ds}}$ facilitates high accuracy measurements of water quality. In addition the calibration of the instrument is also easier due to this linear behavior.

Table 1: Power and statistical Analysis

\begin{tabular}{|c|c|c|c|}
\hline Parameters & PCS & FGMOS & $\begin{array}{c}\text { QFGM } \\
\text { OS }\end{array}$ \\
\hline $\begin{array}{c}\text { CMOS } \\
\text { technology(nm) }\end{array}$ & 70 & 70 & 70 \\
\hline Power supply & $0-1.5 \mathrm{~V}$ & $0-1.5 \mathrm{~V}$ & $0-1.5 \mathrm{~V}$ \\
\hline Average power & $1.43 \times$ & $1.44 \times$ & $1.46 \times$ \\
dissipation & $10^{-3}$ & $10^{-3}$ & $10^{-3}$ \\
\hline Max power & $2.40 \times$ & $2.41 \times$ & $2.42 \times$ \\
& $10^{-3}$ & $10^{-3}$ & $10^{-3}$ \\
\hline Min power & $8.987 \times$ & $8.989 \times$ & $8.99 \times$ \\
& $10^{-10}$ & $10^{-10}$ & $10^{-10}$ \\
\hline Multiple R & 0.983 & 0.987 & 0.990 \\
\hline R Square & 0.966 & 0.971 & 0.981 \\
\hline Standard Error & 0.026 & 0.023 & 0.020 \\
\hline
\end{tabular}

In this design, the device is free from channel length modulation various analyses on the given device reveal that the device has fairly good performance. Power analysis on tanner tool version 15 shows that the device consumes very low power in order of $2.4 \mathrm{~mW}$ as shown in Table 1. The slew rate of the device is good. The output observed in Fig. 4 is highly linear, indicating that the device is stable. The output of the device is obtained and statistical analysis has been done. The various parameters are tabulated in the Table 1 . Coefficient of Determination $\mathrm{R}^{2}$ is found to be $98.1 \%$ with standard error of 0.020 .

\section{Conclusion}

A significant advantage of the proposed design is a simple architecture, and hence it is very suitable for water quality monitoring applications. This study can be extended and more improvement in terms of power and size can be achieved at wiring and layout characteristics level and more effective results can be obtained.

\section{Acknowledgments}

I am very thankful to Dr. Surendra Kumar (BARC Scientist) and Dr. Mirja Tariq Beg (JMI)for their valuable comments on this study time by time. 


\section{References}

[1] L.L.W. Chow et al, "Reactive sputtered $\mathrm{TiO} 2$ thin film humidity sensor with negative substrate bias." Sensors and Actuators B-Chemical, Vol: 76, pp:310-315, 2001.

[2] Whig and S. N. Ahmad, Simulation of linear dynamic macro model of photo catalytic sensor in SPICE, Compel the Int. J. Comput. Math. Electric. Electron. Eng. Vol.: 33 , pp: 611-629, 2013.

[3] Polycarpou, M.M. Uber, J.G. Zhong Wang Feng Shang Brdys, M. "Feedback control of water quality." Control Systems Magazine, IEEE Vol: 22 ,Issue:3 ,pp :68-87, 2002.

[4] P. Whig and S. N. Ahmad, "Development of Economical ASIC For PCS For Water Quality Monitoring", JCSC Vol. 23, No. 6, 2014.

[5] D. Kahng and S.M. Sze, "A floating-gate and its application to memory devices," The Bell System Technical Journal, Vol. 46, No. 4 , pp. 1288-1295, 1987.

[6] P Whig, S.N. Ahmad, "Performance analysis of various readout circuits for monitoring quality of water using analog integrated circuits," International Journal of Intelligent Systems and Applications Vol. 4, pp. 103-109, 2015.

[7] T. S. Lande, D. T. Wisland, T. Saether, and Y. Berg, "FLOGIC-floating-gate logic for low-power operation," in Proceedings of the 3rd IEEE International Conference on Electronics, Circuits, and Systems (ICECS '96), pp. 1041-1044, October 1996.

[8] E. Rodriguez-Villegas, Low Power and Low Voltage Circuit Design with the FGMOS Transistor, vol. 20 of IET Circuits, Devices \& Systems Series, The Institution of Engineering and Technology, London, UK, 2006.

[9] Massobrio, G. and Antognetti, P. (1993), Semiconductor Device Modeling with SPICE, McGraw-Hill, New York, NY.

[10] Kim, Y.-C., Sasaki, S., Yano, K., Ikebukuro, K., Hashimoto, K. and Karube, I.,"Photocatalytic sensor for the determination of chemical oxygen demand using flow injection analysis", Analytica Chimica Acta, Vol. 432 No. 2, pp. 59-66, 2001.

[11] P. Whig and S. N. Ahmad, "Performance analysis of various readout circuits for monitoring quality of water using analog integrated circuits," International Journal of Intelligent Systems and Applications, Vol. 11, pp. 91-98, 2012.

[12] P. Whig and S. N. Ahmad, "Novel FGMOS based PCS device for low power applications," Photonic Sensors Vol. 5, pp. 123-127, 2015. 